\title{
El papel de los anti-inflamatorios no esteroideos en la producción de fasceítis necrotizante
}

\author{
Bertrand Behm-López, ${ }^{1}$ Isaías Salas-Herrera, ${ }^{2}$
}

\begin{abstract}
Resumen: La fasceítis necrotizante es una infección que afecta tejidos blandos, producida por estreptococos beta hemolíticos grupo A. Su diagnóstico temprano es difícil, siendo más fácil conforme avanza el curso de la enfermedad. Para determinar si existía una relación entre los anti-inflamatorios no esteroideos y la aparición de fasceítis necrotizante, fue analizada la literatura médica de estudios clínicos en los últimos 41 años (base de datos de MEDLINE), los cuales evidenciaron que el tamaño de las muestras era pequeño, no eran randomizadas, no tenían grupos control y no lograron demostrar evidencia científica de causa-efecto entre los anti-inflamatorios no esteroideos y la fasceítis necrotizante, sin importar las vías de administración utilizadas. Con el fin de determinar si existe una asociación entre los anti-inflamatorios no esteroideos y fasceítis necrotizante, deben llevarse a cabo en el futuro estudios clínicos bien diseñados.
\end{abstract}

Descriptores: fasceítis necrotizante, anti-inflamatorios no esteroideos, infección, inmunosupresión.

Recibido: 18 de setiembre, 2001

Aceptado para publicación: 21 de diciembre, 2001

Desde los años 80 ha existido a nivel mundial, un aumento significativo de infecciones por Estreptococos ß hemolíticos del Grupo A (EBHGA), las cuales han ocasionado una serie de patologías, entre ellas la fasceítis necrotizante ${ }^{1}$.

En nuestro país, los casos recientes de fasceítis necrotizante generaron informes sensacionalistas sobre su incidencia, transmisión de persona a persona, resistencia al uso de antibióticos y especialmente la supuesta relación entre el uso intramuscular de anti-inflamatorios no esteroideos (AINES) y la fasceítis necrotizante (FN) ${ }^{2}$.
Abreviaturas: Anti-inflamatorios no esteroideos (AINES), Centro de Prevención y Control de Enfermedades (CDC), estreptococos grupo $A b$ hemolíticas (EBHGA), factor mitogenético $F(F M F)$, factor de necrosis tumoral-a (FNT) fasceítis necrotizante (FN), interleucina-1 (IL-1), interleucina-6 (IL -6), sistema de reportes espontáneos (SRS),

M édico A sistente, Centro Nacional de Control del Dolor y Cuidados Paliativos.

Director M édico, Centro Nacional de Control del Dolor y Cuidados Paliativos.

Correspondencia: Dr. Bertrand Behm López, A pdo. 7484-1000, San J osé, FAX: 296-50-69, e-mail: behmlo@ hotmail.com
El objetivo de este artículo fue realizar una revisión de la literatura médica disponible, para determinar si existe evidencia científica que sustente una asociación entre el uso de A INES en sus diferentes presentaciones y la producción de fasceítis necrotizante. También se discutirán posibles mecanismos bioquímicos involucrados en la producción de FN ${ }^{3}$.

En esta revisión científica se utilizó la base de datos de MEDLINE, empleando las siguientes palabras claves: "necrotizing fasciitis" y "non-steroidal anti-inflammatory drugs". Se revisó la literarura médica correspondiente, desde 1952 hasta el año en curso.

\section{Fasceítis Necrotizante}

La FN como entidad nosológica fue descrita por primera vez por Wilson en $1952^{4}$, aunque ya desde la antigüedad (siglo $5^{\circ}$ AC) se cuenta con descripciones, por parte de Hipócrates, de cuadros clínicos aparatosos que muy probablemente son atribuibles a FN ${ }^{5,6}$.

La FN se caracteriza por: 1) ser una infección bacteriana de tejidos blandos, incluyendo grasa subcutánea, fascia superficial y fascia superficial profunda ${ }^{7}$. 2) Clínicamente puede afectar cualquier parte del cuerpo, pero suele ocurrir en alguna de las extremidades ${ }^{8}$. 3) Inicia con presentación de eritema y edema doloroso localizado, con induración importante al rededor del área afectada; además progresa en horas 0, más frecuentemente, entre 2 y 4 días desarrollando cianosis, 
lesiones ampollosas y necrosis ${ }^{9}$. 4) Síntomas y signos sistémicos incluyen fiebre de inicio temprano, ansiedad, estado mental alterado, taquipnea y taquicardia generalmente acompañados por hipocal cemia y aumento importante en los niveles de creatinin fosfoquinasa ${ }^{9}$. 5) La aparición de hipocalcemia y el aumento en los niveles de creatinin fosfoquinasa representan una rápida progresión de signos y síntomas, asociada con una alta tasa de toxicidad sistémica y mortalidad ${ }^{9}$.

La FN se puede clasificar en dos entidades bacteriológicas separadas: a) FN tipo I, que tiene como factor etiológico la presencia de al menos una especie de bacteria anaeróbica, comúnmente Bacteroides y Peptostreptococcus sp. b) FN tipo II, en la que se aíslan EBHGA, ya sean solos, o en combinación con otras bacterias, frecuentemente Staphylococcus aureus ${ }^{10}$. La FN tipo II, denominada también gangrena hemolítica estreptococócica, es la entidad que ha cobrado gran interés epidemiológico en el nivel mundial debido al aumento en incidencia de casos de infecciones por EBHGA y a su alta mortal idad ${ }^{11}$.

Los informes más recientes publicados en Norteamérica sobre tasas de incidencia de FN por EBHGA, oscilan entre 1,7 a 7,0 por 10.000 personas, anualmente ${ }^{12,13,14,15}$. Sin embargo, el Centro de Prevención y Control de Enfermedades (CDC) de A tlanta, estima la incidencia global de F $\mathrm{N}$ en Estados Unidos de Norteamérica entre 4 y 5 casos por cada 100.000 personas por año ${ }^{16}$.

L a mayoría de los casos se presentan en niños menores de 10 años y adultos mayores. Las infecciones invasivas severas ocurren en gente mayor con problemas médicos de fondo diabetes mellitus, enfermedad vascular periférica, nefropatía, hepatopatía crónica. Las tasas de mortalidad en adultos se reportan entre 30 y un $80 \%$, mientras que en estudios epidemiológicos pediátricos se documentan tasas de mortalidad entre un 4 y un $5 \%^{16,17,18}$. Según el estudio de vigilancia poblacional realizado por el CDC, se encontró que el $85 \%$ de las infecciones ocurren espontáneamente en la comunidad, un $10 \%$ son adquiridas en el hospital, un $4 \%$ en instituciones de atención al adulto mayor, tipo asi los de ancianos y solamente un $1 \%$ posterior a contactos estrechos con el paciente portador de $\mathrm{FN}{ }^{16}$.

\section{Mecanismos asociados en el origen de la fasceítis necrotizante}

En las infecciones por EB HGA invasivas se ha asociado a la presencia de proteínas de superficie M-1 y M-3 19,20,21. Otro mecanismo que se víncula con el desarrollo de esta enfermedad es la producción de exotoxinas pirogénicas estreptococócicas, específicamente dos de reciente descubrimiento: factor mitogenético $F(F M F)$ y superantígeno estreptococócico 22,23,24.

También existe activación a gran escala de células $T$ y macrófagos mediada por superantígenos, que conlleva una masiva producción de citoquinas, especialmente de factor de necrosis tumoral (FNT), interleucina-1 (IL-1) e interleucina6 (IL -6). Estas citoquinas son capaces de producir shock y lesión tisular importante ${ }^{19}$. Otros factores que permiten el desencadenamiento de esta patología son los que se presentan en el huésped: la ausencia de anticuerpos antiestreptococócicos y antitoxinas de tipo específico (debido a falta de exposición previa a cepas más virulentas, portadoras de proteínas de superficie M-1 y M-3) o la presencia de una enfermedad debilitante 0 inmunosupresora de fondo 19,25,26.

Los mecanismos patogenéticos anteriormente mencionados llevaron al Dr. Dennis L. Stevens (1995), a postular un hipotético mecanismo bioquímico que establece la posible asociación del uso de AINES con el desarrollo de infecciones invasivas por EBHGA 4 .

Los A INES, además de su efecto anti-inflamatorio, se les ha asociado con efectos secundarios como: a) la supresión de funciones granulocíticas, como lo son: quimiotaxis, fagocitosis, actividad oxidativa y destrucción bacteriana, las cuales inicialmente podrían provocar que infecciones cutáneas menores progresen a fulminantes, b) en relación con estos efectos secundarios, los AINES podrían permitir la circulación excesiva de FNT al bloquearse la síntesis de $\mathrm{PgE}_{2}{ }^{4,27,29}$. Como se mencionó anteriormente, los nivel es elevados circulantes de FNT conllevan a lesiones tisulares importantes ${ }^{19}$.

\section{Evidencias clínicas}

El primer informe oficial en el que se menciona la asociación del uso de AINES y FN aparece en 1996 en él Solomon documenta la reactivación de un cuadro infeccioso latente posterior al uso de AINEs por vía oral. Este estudio fue producto de la observación de un solo caso clínico ${ }^{30}$.

En 1978, estudios in vitro por Solberg et al ${ }^{31}$, indican que los A INES podrían interrumpir el normal funcionamiento de los granulocitos, los cuales al ser encubados con fenilbutazona presentan una depresión de su cualidad bactericida y una reducida inactivación de cepas de Staphylococcus aureus y estreptococos grupo B.

En 1985 B run-Buisson et al ${ }^{32}$ reportan un estudio seriado, re trospectivo, no randomizado, doble-ciego, ni cruzado, de 31 pacientes con FN hospitalizados en $\mathrm{H}$ enri Mondor de Paris. De los 31 pacientes, solo 6 no presentaban una patología debilitante de fondo. Cada uno de estos 6 pacientes, previamente sanos, había recibido tratamiento a dosis terapéuticas con A INES vía oral, por condiciones inflamatorias no especificadas o por flebitis superficial. De los 6 pacientes, 4 presentaban historia de trauma menor y en todos los casos excepto por uno, se aislaron cultivos positivos por EB HGA. Este estudio utilizó una muestra muy pequeña, no randomizada, ni controlada, por lo que no se pudo establecer conclusiones estadísticamente significativas, solo se obtuvieron evidencias observacionales.

K rige et al ${ }^{33}$ (1985) describen el caso de un hombre de raza caucásica, de 23 años, quien ingiere 6 tabletas de diflunisal 
$(1500 \mathrm{mg}$ ) en un periodo de 24 horas, por un cuadro de gonartralgia posterior a un trauma en rodilla, acompañado de una escoriación mínima. Inicialmente se trató como un caso de celulitis. Sin embargo, posterior a un deterioro clínico trepidante y una cirugía exploratoria, se llega al diagnóstico de FN. La secreción purulenta aislada del espacio articular cultivó EBHGA. Luego, el grupo de Krige realiza un estudio comparativo, en el cual se analiza la capacidad linfocítica en la conversión de fitohemaglutinina ( $\mathrm{FHA}$ ) en los linfocitos del paciente y en linfocitos de un grupo control. Ese estudio demostró que las células control no presentaron ninguna alteración, mientras que los linfocitos del paciente presentaron un $22 \%$ de depresión en la conversión de FHAy un $14 \%$ de depresión en su tasa de transformación. Este hallazgo observacional, se basa en solo un paciente, por lo que no es posible inferir datos significativos.

En un estudio similar, Espersen y Larsen ${ }^{34}$ logran determinar en leucocitos de una paciente de 32 años con $\mathrm{FN}$, alteraciones en la función de quimiotaxis en neutrófilos, así como de quimioluminicencia y transformación linfocítica de FHA, posterior a recibir tratamiento con AINES por vía oral. Sin embargo, nuevamente el estudio se realiza con datos obtenidos en un solo paciente.

A su vez, Rimailho et al ${ }^{35}$ realizan una revisión retrospectiva de casos de $\mathrm{FN}$ en K remlin-Bicetre, Francia, durante los años de 1982 a 1985. Se encontraron 7 casos de FN, de los cuales 5 ocurrieron en pacientes previamente sanos. De estos 5 pacientes, 4 habían recibido tratamiento con A INES por vía oral y 1 se encontraba recibiendo $2 \mathrm{~g}$ de A A S diario; medicamentos que fueron prescritos por diagnósticos preliminares de artritis, flebitis y linfedema. De nuevo en todos los casos, excepto en uno, se aislaron cepas de EBHGA (en este caso, al igual que en la serie descrita por $B$ run-B uisson, los pacientes con cultivos negativos habían recibido tratamiento con penicilina 24 horas antes de ser admitidos en los centros de salud).

También en este estudio la muestra es pequeña y no hubo un estudio control.

A simismo, Smith y Berk ${ }^{36}$ reportan el caso de un paciente masculino de 31 años, previamente sano, quien posterior a la extracción traumática de un diente bajo condiciones sépticas (con un alicate, realizada por familiares del paciente) recibe piroxicam debido al desarrollo de un aparente dolor muscular en su hombro izquierdo. De acuerdo con la historia clínica, el paciente ingirió 8 comprimidos de $10 \mathrm{mg}$ de fuerza cada uno, en un periodo de aproximadamente 24 horas, tras lo cual desarrolló un cuadro aparatoso de $\mathrm{FN}$ a nivel de miembro superior derecho y murió 17 días después de su ingreso al centro hospitalario. Nuevamente cultivos aislados de la zona afectada resultan positivos por EB HGA. Al igual que en los casos anteriores, no se logra llegar a ninguna conclusión que tenga confiabilidad estadística.

En casos otorrinolaringológicos de $\mathrm{FN}$ también se ha encontrado una aparente asociación entre $\mathrm{FN}$ y el uso de AINES.
Chaplain ${ }^{37}$ documenta 20 casos de $\mathrm{FN}$ cervical aguda tratados entre 1986 y 1995, de los cuales 5 se definieron como de origen faríngeo. Entre estos 5 pacientes, 4 habían recibido esteroides 0 anti-inflamatorios no esteroideos por vía oral. No se presentó ninguna muerte; sin embargo, todos los pacientes requirieron como mínimo 30 días de intubación endotraqueal y terapia con oxígeno hiperbárico. Dada la experiencia clínica, el autor recomienda evitar la utilización de tratamiento anti-inflamatorio esteroideo y no esteroideo en casos de faringitis infecciosa y optar por otros tipos de fármacos antálgicos. Este estudio es de una muestra muy pequeña y carece de un grupo control.

En pacientes pediátricos se realizó un estudio de casos-control, evaluándose la asociación entre $\mathrm{FN}$ y el uso de ibuprofeno ante la presencia de varicela ${ }^{38}$. En el estudio se compararon los casos de 19 niños con varicela y FN con un grupo control constituido por 29 pacientes que presentaban infecciones de tejidos blandos severas, diferentes a FN, acompañando al cuadro de varicela. En los casos de FN se demostró una probabilidad 5 veces mayor de haber recibido tratamiento con ibuprofeno vía oral, previo a su hospitalización, que en niños del grupo control (IC 95\% 1.03-26.6).

Luego de corregir por aislamiento de EBHGA, edad y sexo, la tasa de probabilidad aumentó a 10.2 (IC 95\% 1.3-79.5). $\mathrm{N}$ o se encontró ninguna diferencia estadísticamente significativa cuando se analizó la presencia concomitante de otros posibles factores de riesgo como: uso de difenhidramina, loción de cal amina, condiciones médicas preexistentes, asistencia a guarderías y duración de síntomas de infección secundaria antes de la hospitalización. Solo en cuanto al uso de ibuprofeno se demuestra una diferencia significativa entre ambos grupos ( $42 \%$ vrs. $23 \%, P=0.02)$, la cual estadísticamente hablando, sería muy improbable de atribuir a una asociación aleatoria.

De manera más reciente, en una revisión del sistema de reportes espontáneos (SRS) de la Oficina de A dministración de Fármacos y A limentos en Estados Unidos de N orteamérica ${ }^{39}$, analizaron 33 casos de $\mathrm{FN}, 10$ de los cuales resultaron en el deceso del paciente. En la mayoría de los casos se informa el uso concomitante de A INES para el tratamiento de al guna patología aguda, como varicela, trauma, dolor postoperatorio. De acuerdo con los autores, el número de casos encontrados por medio del SRS no sugiere que la incidencia de FN sea mayor cuando se asocia al uso de AINES o su desarrollo más frecuente sin la presencia de otros factores predisponentes. Conclusiones similares fueron emitidas por la Academia A mericana de Pediatras en Estados U nidos ${ }^{40}$, quienes consideraron que de acuerdo a los datos y estudios realizados hasta el momento, no existe evidencia contundente para eliminar en nivel institucional el uso de AINES en casos de varicela. Sin embargo, sí hacen énfasis en utilizar AINES en estos casos bajo controles clínicos estrictos.

En un revisión de FN similar, realizada en adultos, Green et al 41 argumentan que dado el uso tan amplio y generalizado de AINES para todo tipo de dolores en casos de lesiones 
cutáneas, faringitis y traumas menores, se podrían teóricamente encontrar no solo millones, sino billones de situaciones clínicas, en que el uso de AINES no ha predispuesto una infección por EB HGA severa como FN. Por lo tanto, concluyen que el tipo de asociación entre ambas entidades es más bien de simple correlación y no de causa-efecto.

Finalmente, en el más reciente estudio de casos-control realizado en Ontario, Canadá 42 , entre los años de 1991 y 1996, en un total de 524 casos estudiados, de pacientes con infecciones de tejidos blandos por EBHGA, dentro de una población total de 1080 casos, se concluyó que el uso de A INES no predisponía a pacientes con enfermedades crónicas a desarrollar FN como complicación en comparación con otros pacientes. Sin embargo, mencionan que aquellos que iniciaron el tratamiento con AINES antes del inicio de alguna patología crónica, sí presentaban una mayor incidencia de FN como complicación.

\section{Concluciones}

La FN es una entidad nosológica cuya incidencia y consecuente mortalidad ha aumentado en el nivel mundial en años recientes. Dicha enfermedad es frecuentemente difícil de diagnosticar en sus estadios tempranos, cuando los cambios cutáneos no han progresado.

L a mayoría de los estudios clínicos presentes en la literatura médica de los últimos 50 años, fueron realizados con muestras muy pequeñas, no randomizadas y sin estudios control, por lo que no permiten obtener conclusiones significativas que pudieran establecer una relación causal entre el uso de A INES y la presencia de FN. Este argumento es respaldado por $\mathrm{G}$ reen et al ${ }^{41}$, quienes mencionan que el número de prescripciones de AINES en sus diferentes presentaciones es de millones 0 inclusive billones en Estados Unidos de N orteamérica. Estas drogas se administran a personas sanas que cursan con lesiones cutáneas, traumas menores, procesos inflamatorios, teniendo una incidencia de únicamente 4 a 5 casos por cada 100.000 habitantes anual mente ${ }^{14}$, incidencia muy baja para el uso tan generalizado que se realiza de estos medicamentos. Sí es importante resaltar el cuidado especial que se debe tener al prescribirlos a poblaciones de alto riesgo, constituidas por pacientes inmunosuprimidos y portadores de infecciones severas.

Tampoco se encontró evidencia sustentada por estudios científicos, reproducibles, que muestren que la FN se presente con mayor frecuencia cuando se administran A INES a través de la vía parenteral ${ }^{38,39}$, como se ha mencionado en estudios aislados ${ }^{2}$. En resumen, no existe base científica para prohibir en forma universal el uso de AINES.

Como principal arma de defensa para combatir los episodios de FN, recomendamos ante todo la prevención de la patología, la cual únicamente puede lograrse a través de la educación del personal de los centros de salud de los pacientes y sus familiares. Se debe enfatizar en un adecuado método de higiene (lavado de manos, evitar compartir utensilios de higiene personal, como cepillos de dientes); refuerzo de la vigilancia epidemiológica hospitalaria, especialmente en los procedimientos quirúrgicos, de obstetricia y de manejo de catéteres intravenosos; el control estricto de visitas por paciente hospitalizado; el control de basura y excretas de la comunidad y charlas informativas al público en general. También se debe tener especial cuidado en el manejo de heridas contaminadas y sitios de inyección en drogadictos. A demás, en el seguimiento de los pacientes debemos tomar en cuenta posibles factores de riesgo para el desarrollo de $\mathrm{FN}$ al prescribir A INES, como lo son: edad avanzada, alcoholismo, varicela en niños, enfermedad vascular periférica, hepatopatías y nefropatías crónicas, así como el síndrome de inmunodeficiencia adquirida ${ }^{40}$.

\section{Summary}

Necrotizing fasceiitis, as a severe group A streptococcus infection generally originating from localized skin infections or disruptions has increased worldwide in recent years. Early diagnosis is often difficult due to the appearance of characteristic soft tissue changes only late in the course of the disease. The medical literature was analized to determine a possible association between non-setroidal antiinflammatory drugs and necrotizing fasceiitis and possible factors involved in necrotizing fasceiitis. Sample sizes of the clinical studies reviewed were small, non-randomized, without control groups. Thus, no clinical studies showed scientific evidence of a cause and effect relationship between non-setroidal anti-inflammatory drugs and necrotizing fasceiitis, regardless of the route of administration. Further well-designed clinical trials should be carried out to determine if there is a possible association between nonsetroidal anti-inflammatory drugs and necrotizing fasceítis.

\section{Referencias}

1. Bisno A, Stevens D. Streptococcal infections of skin and soft tissues. N Engl J M ed 1996; 334:240.

2. Guy C. Fulminating necrotizing fasciitis after intramuscular injection of a corticoid. Contributing role of non-steroidal anti-inflammatory agents. Therapie. 1993; 48: 489-90.

3. Stevens DL, Tanner M H, Winship J, Swarts R; Ries KM, Schlievert $P M, K$ aplan E. Severe group A streptococcal infections associated with a toxic shock-like syndrome and scarlet fever toxin A. N Engl J Med 1989; 321:1-7.

4. Wilson CB. Necrotizing fasciitis. A m Surg 1952; 18:416.

5. Stevens DL. Could nonsteroidal anti-inflammatory drugs (NSAID) enhance de progression of bacterial infections to toxic shock syndrome? Clin Infect Dis 1995; 21: 977.

6. Brantigan CO, Senkowsky J. Group A beta hemolytic streptococcal necrotizing fasciitis. Wounds 1995; 7:62-8.

7. Descamps V, Aitken J, Lee M G. Hippocrates on necrotising fasciitis. L ancet 1994; 344:556. 
8. Kahn LH. Necrotizing soft tissue infections reported with nonsteroidal antiinflammatory drugs. A nn Pharmacother 1997; 31(9): 1034-9.

9. Kotrappa KS, Bansal RS, A min NM. Necrotizing Fasciitis. Am Fam Physician 1996; 53:1691-7.

10. Chapnick EL, A bter El. Infectious Disease Emergencies- Necrotizing soft tissue infections. Infect Dis Clin N orth A m 1996; 10(4): 835-55.

11. Bergdoll MS, Chesney PJ. Toxic Shock Syndrome. Boca Raton, FL, CRC Press, 1991.

12. M orantes M, Lipsky BA. "Flesh eating bacteria": return of an old nemesis. Int J Dematol 1995; 34:461-3.

13. Katz $A R, M$ orens $D M$. Severe streptococcal infections in historical perspective. Clin Infect Dis 1992; 14:298-307.

14. Hoge CW, Schwartz B, Talkington DF, B reiman RF, M acN eill EM, Englender $\mathrm{SJ}$. The changing epidemiology of invasive group $\mathrm{A}$ streptococcal infections and the emergence of streptococcal toxic shock-like syndrome: a retrospective population-based study. J A M A 1993; 269:384389.

15. Davies HD, M CGeer A, Schwartz B, Green K, Cann D, Simor A E, L ow DE. Invasive group A streptococcal infections in Ontario, Canada. N Engl J M ed 1996;335:547-554.

16. Centers for Disease Control and Prevention. Group A beta-hemolytic streptococcal bacteremia: Colorado, 1989. M M WR 1990;39:3-6.

17. O'B rien $K L$, L evine $O S$, Schwartz B. The changing epidemiology of group A streptococcus infections. Semin Pediatr Infect Dis. 1997; 8:1-8.

18. Davies HD, Matlow A, Scriver SR, Lovgren M, Talbot JA, Low DE. A pparent lower rates of streptococcal toxic shock syndrome and lower mortality in children with invasive group A streptococcal infections compared with adults. Pediatr Infect Dis J 1994;13:49-56.

19. Kaplan EL. Recent epidemiology of group A streptococcal infections in North A merica and abroad: an overview. Pediatrics 1996; $97(6$ Pt 2): $945-8$

20. Cockerill FR, M acD onald KL, Thompson RL, Roberson F, K ohner PC, B esser-W iek J, et al. A n outbreak of invasive group A streptococcal disease associated with high carriage rates of the invasive clone among school-aged children. J A M A 1997; 277:38-43.

21. Wolf JE, Rabinowitz LG. Streptococcal toxic shock-like syndrome. A rch Dermatol 1995;131:73-7.

22. Bronze MS, Dale JB. The reemergence of serious group A streptococcal infections and acute rheumatic fever. A m J M ed Sci 1996; 311:4154.

23. Stevens DL, Tanner M H, Winship J, Swarts R, Ries KM, Schlievert $P M$, et al. Severe group A streptococcal infections associated with a toxic shock-like syndrome and scarlet fever toxin A . N Engl J M ed 1989; 321:1-7.

24. Schwartz B, Facklam RR, Breiman RF. Changing epidemiology of group A streptococcal infection in the USA . Lancet 1990;336:1167-71.

25. White J, Herman A, Pullen A M , K ubo R, K appler JW, M arrack P. The $V$ beta-specific superantigen staphylococcal enterotoxin $B$ : stimulation of mature Tcells and clonal deletion in neonatal mice. Cell 1989;56:27-35.

26. L eung DY M , Travers J B, N orris DA. The role of superantigens in skin disease. J Invest Dermatol 1995;105(Suppl):37s-42s.

27. Rosen H. Superantigens. Int J Dermatol 1997;36:14-6. Schlievert PM . Role of superantigens in human disease. J Infect Dis 1993;167:9971002.
28. Shimozato T, I wata M, Tamura N. Suppression of tumor necrosis factor al pha production by a human immunoglobulin preparation for intravenous use. Infect Immun 1990; 58:1384.

29. Stevens $D L, B$ ryant $A E$, Hackett $S P, C$ hang $A$, Peer $G, K$ osanke $S$, Emerson T, Hinshaw L. Group A streptococcal bacteremia: The role of tumor necrosis factor in shock and organ failure. J Infect Dis 1995; 173:619-626

30. Solomon L. A ctivation of latent infection by indomethacin: a report of three cases. Br. M ed. J 1996; 1:961-62.

31. Solberg CO, Alfred CD, Hill HR. Influence of phenylbutazone on leukocyte chemiluminescence and function. A cta Pathol M icrobiol Immunol Scand [C] 1978; 86:165-71

32. B run-Buisson CJ, Saada M, Trunet P, Rapin M, Roujeau JC, Revuz J Haemolytic streptococcal gangrene and non-steroidal-anti-inflammatory drugs. Br M ed J 1985; 290:1786.

33. K rige JE, Spence RA, Potter PC, Terblanche J. Necrotizing Fasciitis after diflunisal for minor injury. Lancet 1985; 2: 1432-1433.

34. Espersen GT, Larsen K. NSAID and fasciitis necroticans. U geskr Laeger 1987; 145:3475-3477.

35. Rimailho A, Riou B, Richard C, A uzepy P. Fulminant necrotizing fasciitis and non-steroidal-antiinflammatory drugs. J Infect Dis 1987; 155:143-147.

36. Smith RJ, Berk SL. Necrotizing fasciitis and Nonsteroidal A nti-inflammatory Drugs. South M ed J. 1991; 84: 785-787.

37. Chaplain A. A cute cervical necrotizing fasciitis of pharyngeal origin: possible role of steroidal and non-steroidal anti-inflammatory agents. A propos of 5 cases. Rev Laryngol Otol Rhinol (Bord) 1996; 117(5): 377-80.

38. Zerr DM, A lexander ER, Duchin J S, K outsky LA, Rubens CE. A casecontrol study of necrotizing fasciitis during primary varicella. Pediatrics 1999; 103:783-90.

39. K ahn LH. N ecrotizing soft tissue infections reported with non-steroidal antiinflammatory drugs. A nn Pharmacother 1997; Sep; 31(9): 1034-9.

40. Severe Invasive Group A Streptococcal Infections: A Subject Review (RE9804) A merican A cademy of Pediatrics Committee on Infectious Diseases, 1999

41. Green RJ, Dafoe DC, Raffin TA . Necrotizing Fasciitis. Chest 1996; 110 (1): 1

42. Canadian Bacterial Surveillance N etwork, December 1999

43. Stone DR, Gorbach SL. Necrotizing fasciitis. The changing spectrum. Dermatol Clin 1997; 15(2): 213-20. 